\title{
Developmental Origin of the Neuronal Subtypes That Comprise the Amygdalar Fear Circuit in the Mouse
}

\author{
Ronald R. Waclaw, ${ }^{1}$ Lisa A. Ehrman, ${ }^{1}$ Alessandra Pierani, ${ }^{2}$ and Kenneth Campbell ${ }^{1}$ \\ ${ }^{1}$ Division of Developmental Biology, Cincinnati Children's Hospital Medical Center, University of Cincinnati College of Medicine, Cincinnati, Ohio 45229, \\ and 2 Program in Development and Neurobiology, Institut Jacques Monod, Centre National de la Recherche Scientifique Unité Mixte de Recherche 7592, \\ Université Paris Diderot, 75013 Paris, France
}

We have taken a genetic-based fate-mapping approach to determine the specific contributions of telencephalic progenitors to the structures that comprise the amygdalar fear circuit including the central (CA), lateral (LA), and basolateral (BLA) amygdala. Our data indicate that progenitors in the ventral pallium (VP) contribute projection neurons to the LA and BLA but not the CA. Rather, the CA appears to derive, at least in part, from progenitors located in the ventral lateral ganglionic eminence (vLGE). Diverse groups of interneurons populate these amygdalar nuclei, and as predicted our data support the notion that they originate from subpallial progenitors. A rather specific population of amygdalar interneurons, the intercalated cells (ITCs), is known to play a fundamental role in fear-related behaviors. However, no information on their specific origin has, as yet, been provided. Our findings suggest that the ITCs arise from the dorsal lateral ganglionic eminence (dLGE) and migrate in the lateral migratory stream to populate the paracapsular regions as well as the main intercalated mass of the amygdala (IA). Germ-line Gsx2 mutants are known to exhibit an expansion of the VP into the LGE and a concomitant reduction in the dLGE and vLGE. Accordingly, Gs 2 conditional mutants display a significantly enlarged LA and a significant reduction in ITCs both within the paracapsular regions and the IA. Additional support for a dLGE origin of the ITCs was obtained in conditional mutants of the dLGE gene Sp8. Thus, our findings indicate diverse origins for the neuronal components that comprise the amygdalar fear circuit.

\section{Introduction}

The mammalian amygdala is composed of a large number of distinct nuclei, which collectively control the emotional state of the animal (Swanson and Petrovich, 1998). A major function of the amygdala is to control fear- and anxiety-related behaviors in cooperation with the cerebral cortex and hippocampus (LeDoux, 2000; Roozendaal et al., 2009). The amygdalar nuclei that control fear responses include the lateral (LA), basolateral (BLA), and the central (CA) amygdala (Paré et al., 2004; Ehrlich et al., 2009). The LA and BLA represent the input to the amygdala and exhibit cortex-like characteristics with a majority of glutamatergic projection neurons (Swanson and Petrovich, 1998). Conversely, the CA contains striatum-like GABAergic projection neurons and represents the major output of the amygdala (McDonald, 1982; Swanson and Petrovich, 1998). Neuronal activity in these nuclei is modulated by diverse sets of inhibitory interneurons. In particular, the intercalated cells (ITCs), which are clustered along paracapsular regions of the LA and BLA and in the main interca-

Received Nov. 19, 2009; revised March 5, 2010; accepted April 5, 2010.

This work was supported by National Institutes of Health (NIH) Grant NS044080. R.R.W. is supported by NIH Training Grant HD046387. We thank T. Jessell for providing the /s/1-cre mice, S. Anderson and E. Lai for providing the Foxg $7^{\text {tTA }}$ mice, M. C. Colbert for providing the CC-EGFP mice, as well as A. Nagy and J. Whitsett for providing the tet0-cre mice. We are also grateful to A. Buchberg, P. Emson, S. Morton, and T. Jessell for providing antibodies.

Correspondence should be addressed to Kenneth Campbell, Division of Developmental Biology, Cincinnati Children's Hospital Medical Center, University of Cincinnati College of Medicine, 3333 Burnet Avenue, Cincinnati, $\mathrm{OH}$ 45229. E-mail: kenneth.campbell@cchmc.org.

DOI:10.1523/JNEUROSCI.5772-09.2010

Copyright $\odot 2010$ the authors $\quad 0270-6474 / 10 / 306944-10 \$ 15.00 / 0$ lated cell mass of amygdala (IA), have been shown to play important roles in fear extinction (Jüngling et al., 2008; Likhtik et al., 2008). Despite the prominent role that the amygdala plays in emotional control and particularly fear responses, relatively little is known about the development of this telencephalic structure.

Previous studies have suggested that the amygdala derives from progenitors located in both the pallium (dorsal telencephalon) and subpallium (ventral telencephalon) (Swanson and Petrovich, 1998; Medina et al., 2004). The pallial nature of the LA and BLA and subpallial character of the CA is supported by the fact that most GABAergic neurons of the telencephalon derive from the subpallium, whereas glutamatergic neurons appear to originate in the pallium (for review, see Marín and Rubenstein, 2001). The LA and BLA projection neurons have been suggested to derive from the ventral pallium (VP) and lateral pallium, respectively (Medina et al., 2004). More recent studies, however, suggest that the VP can give rise to projection neurons of both the LA and BLA (Stenman et al., 2003b; Hirata et al., 2009). The specific telencephalic progenitor domain that gives rise to the CA has not been clearly defined. Moreover, the GABAergic interneuron subtypes that contribute to the amygdala are presumably derived exclusively from the subpallium. Indeed, genetic fate maps of Nkx2.1expressing [i.e., medial ganglionic eminence (MGE)] progenitors show scattered cells throughout the amygdalar complex including the LA, BLA, and CA (Xu et al., 2008). The developmental origin of the ITCs has not been addressed; however, their morphology and GABAergic nature (Millhouse, 1986; 
Marowsky et al., 2005) suggest that they would originate within the subpallium as well.

We have used genetic fate mapping together with mutant mouse analysis to map the telencephalic progenitor domains that give rise to the neuronal subtypes comprising the amygdalar fear circuit. Our data demonstrate that the neuronal constituents of this circuit derive from distinct and adjacent progenitors domains within the lateral and ventral telencephalon.

\section{Materials and Methods}

Animals. Dbxi $1^{\text {cre/+ }}$ mice (Bielle et al., 2005) were genotyped with the following primers: Jcre5 (5'-GCGGTCTGGCAGTAAAAACTATC-3') and Jcre3 (5'-GTGAAACAGCATTGCTGTCACTT-3') generating a 100 bp product. Dlx5/6-CIE mice were genotyped as previously described (Stenman et al., 2003a). Isl1 ${ }^{\text {cre/+ }}$ mice (Srinivas et al., 2001) were genotyped with the cre primers described above. CagCat(CC)-EGFP were genotyped as described by Nakamura et al. (2006). Gs $x 2^{E G F P /+}$ mice were genotyped as previously described (Wang et al., 2009). CC-EGFP female mice were crossed with male mice from one of the following cre lines, $D b x 1^{\text {cre/+ }}, D l \times 5 / 6-C I E$, or Isl1 ${ }^{\text {cre/+ }}$. Double-transgenic brains were collected between postnatal day 45 (P45) to P60.

$G s \times 2^{f l /+}$ mice and Gs $x 2^{R A /+}$ mice were generated and genotyped as described by Waclaw et al. (2009). Foxg1 ${ }^{t T A /+}$ (Hanashima et al., 2002) mouse embryos and adults were genotyped with the following primers, tTA5 (5'-CGCTGTGGGGCATTTTACTTTAG-3') and tTA3 (5'-CATGTCCAGATCGAAATCGTC-3'), which generates a 500 bp product. Tet-O-cre (Perl et al., 2002) mouse embryos and adults were genotyped with the cre primers described above. Adult mice (between P30 and P90) and mouse embryos [embryonic day 12.5 (E12.5) to E18.5] with targeted deletion of Gs $x 2$ in telencephalon were generated by crossing Foxg ${ }^{t T A /+}$; $G s \times 2^{f l / f l}$ male mice with tetO-cre; $G s x 2^{f l / f l}$ female mice to generate doubletransgenic adult mice and mouse embryos $\left(\right.$ Foxg $\left.1^{t T A /+} ; G s \times 2^{f l f l}\right)$ as controls and triple-transgenic adult mice and mouse embryos (Foxg ${ }^{t T A /+}$; tetO-cre; $\left.G s \times 2^{f l f l}\right)$ as Gsx2 conditional mutants. Mice containing a ventral telencephalon deletion of $S p 8$ (i.e., Dlx5/6-CIE;Sp $8^{f l / f l}$ ) were generated and genotyped as previously described (Waclaw et al., 2006).

For staging of embryos, the morning of vaginal plug detection was designated E0.5. Embryos were fixed overnight in $4 \%$ paraformaldehyde, rinsed thoroughly in PBS, and cryoprotected in $30 \%$ sucrose in PBS before sectioning at $12 \mu \mathrm{m}$ on a cryostat. Postnatal brains were fixed overnight in $4 \%$ paraformaldehyde, rinsed thoroughly in PBS, and cryoprotected in $20 \%$ sucrose in PBS before sectioning at $35 \mu \mathrm{m}$ on a freezing sliding microtome.

Immunohistochemistry. Primary antibodies were used at the following concentrations: rabbit anti-calbindin, 1:4000 (provided by P. Emson, Cambridge University, Cambridge, UK); goat anti-calretinin, 1:2000 (Millipore); goat anti-FoxP2, 1:1500 (Abcam); rabbit anti-FoxP2, 1:5000 (Abcam); goat anti-enhanced green fluorescent protein (EGFP), 1:5000 (Abcam); rabbit anti-EGFP, 1:500 (Invitrogen); rabbit anti-Er81, 1:5000 (provided by S. Morton and T. Jessell, Columbia University, New York, NY); rabbit antiGsx2, 1:4000 (Toresson et al., 2000); rabbit anti-Mef2c, 1:2000 (ProteinTech Group); rabbit anti-Meis2, 1:5000 (provided by A. Buchberg, Thomas Jefferson University, Philadelphia, PA); guinea pig anti- $\mu$-opioid receptor (OR) 1, 1:3000 (Millipore); rabbit anti-serotinin receptor $1 \mathrm{~d}$ (Htr1d), 1:500 (Millipore); rabbit anti-Tbr1, 1:4000 (Millipore). Bright-field staining using diaminobenzidine as the chromogen was done as previously described by Waclaw et al. (2006). The secondary antibodies for fluorescent staining were as follows: donkey anti-goat antibodies conjugated to Cy2, Сy3, or Cy5 (Jackson ImmunoResearch), donkey anti-guinea pig antibodies conjugated to Cy3 or Cy5 (Jackson ImmunoResearch), and donkey anti-rabbit antibodies conjugated to Cy2, Cy3, or Cy5 (Jackson ImmunoResearch). All EGFP stains associated with the Gs $x 2^{E G F P /+}$ knock-in mice were performed using the tyramide amplification kit (Invitrogen; T20932).

Quantification. Quantification of the fate map analysis in $\mathrm{Db} \mathrm{I}^{\mathrm{cre} /+}$; CC-EGFP mice and Dlx5/6-CIE;CC-EGFP mice was done by counting 40-50 EGFP-positive cells in the basolateral complex (BLA and LA) in each of three different animals. $D b x 1$ fate-mapped cells were quantified for Tbr 1 expression, and $D l \times 5 / 6$ fate-mapped cells were quantified for calbindin $(\mathrm{CB})$ expression.

To quantify Gsx2-EGFP/Tbr1 double labeling in controls (Gsx2 $\left.2^{E G F P /+}\right)$ and Gsx2 mutants (Gs $\left.x 2^{E G F P / R A}\right)$, at least 100 EGFP cells were analyzed for Tbr1 expression in the lateral migratory stream for each control $(n=3)$ or Gsx2 mutant $(n=3)$. Statistics were used to compare EGFP/Tbr1positive cells in controls compared with Gsx2 mutants using Student's unpaired $t$ test.

The size of specific amygdalar nuclei was estimated using the NIH ImageJ program. The following markers were used in the analysis of Gs $x 2$ conditional mutants: LA, Mef2c expression; BLA, Er81 expression; CA, Htr1d expression; and IA, Foxp2 expression. Tbr 1 expression was used to analyze the basolateral complex (BLA and LA) in Sp 8 conditional mutants. Briefly, the cross-sectional area of expression for each factor was measured for every relevant brain section in the ImageJ program. The area measurements were averaged based on total number of sections analyzed per brain. Three different controls and Gs $x 2$ conditional mutants or controls and $S p 8$ conditional mutants were used for each experiment. Statistics were performed on Gs $x 2$ conditional mutants compared with controls for the LA, BLA, and CA and for Sp8 conditional mutants compared with controls for the basolateral complex (Tbr1 expression) using Student's unpaired $t$ test. The significance of area differences in the IA of controls, Gs $x 2$ and $S p 8$ conditional mutants was determined using a one-way ANOVA.

To determine the density of cells in the LA and BLA of controls and Gsx2 conditional mutants, Mef2c- or Er81-positive cells were counted in the LA and BLA, respectively, in random areas of the expression domain in at least two sections per animal ( $n=3$ for controls and Gsx2 conditional mutants). These defined areas were then measured in the ImageJ program to determine cells per square millimeter. Statistics were performed on Gsx2 conditional mutants compared with controls using Student's unpaired $t$ test.

To quantify $D b x 1$ fate-mapped cells in E18.5 controls $\left(D b x 1^{\text {cre } /+} ; C C\right.$ EGFP;Gs $\left.\times 2^{+/+}\right)$and germ-line Gs $x 2$ mutants $\left(D b x 1^{\text {crel+ }} ; C C-E G F P\right.$; $\left.G s \times 2^{R A / R A}\right)$, EGFP-positive cells were counted in two different sections of the basolateral complex (BLA and LA) in Dbxi fate-mapped controls $(n=3)$ and $D b x 1$ fate-mapped germ-line Gsx2 mutants $(n=3)$. Total EGFP-positive cells were counted in the LA and BLA. Mef2c/EGFP double-positive cells were counted in the LA and divided by the total EGFP-positive cells in the LA to determine what percentage of $D b x 1$ fate-mapped cells were Mef2c-positive. Statistics were performed on $D b x 1$ fate-mapped germ-line Gsx2 mutants compared with $D b \times 1$ fatemapped controls using Student's unpaired $t$ test.

\section{Results}

\section{Fate mapping amygdalar nuclei}

To determine the developmental origin of neurons in the amygdalar fear circuit, we used cre/loxP fate mapping from distinct progenitor domains of the developing telencephalon with mice expressing cre recombinase in the following progenitor domains of the telencephalon, the VP with $D b x 1^{\text {cre/+ }}$ mice (Bielle et al., 2005), ventral telencephalon, including the MGE, lateral ganglionic eminence (LGE), and caudal ganglionic eminence (CGE) with Dlx5/6-CIE mice (Stenman et al., 2003a), and in the ventral lateral ganglionic eminence (vLGE) (Stenman et al., 2003a) with Isl $1^{\text {cre/+ }}$ mice (Srinivas et al., 2001). Each cre line was crossed with cre reporter CC-EGFP mice (Nakamura et al., 2006) to generate doubletransgenic adult animals that express EGFP after cre recombination.

Distinct patterns of EGFP expression were observed when comparing adult brains with the $D b x 1$ fate map of the VP and Dl $x 5 / 6$ fate map of the ventral telencephalon. Dbx $1^{\text {cre/+ }} ; C C$ EGFP mice expressed EGFP in scattered cells of the LA, BLA, basomedial and medial amygdala (MA), as well as in the pyriform cortex (Fig. 1A) (Hirata et al., 2009). Dlx5/6-CIE;CC-EGFP mice exhibited a more widespread recombination pattern with many EGFP-positive cells in the CA, MA, and IA (Fig. 1D). Additionally, scattered Dlx5/6 fate-mapped cells were observed in the LA 

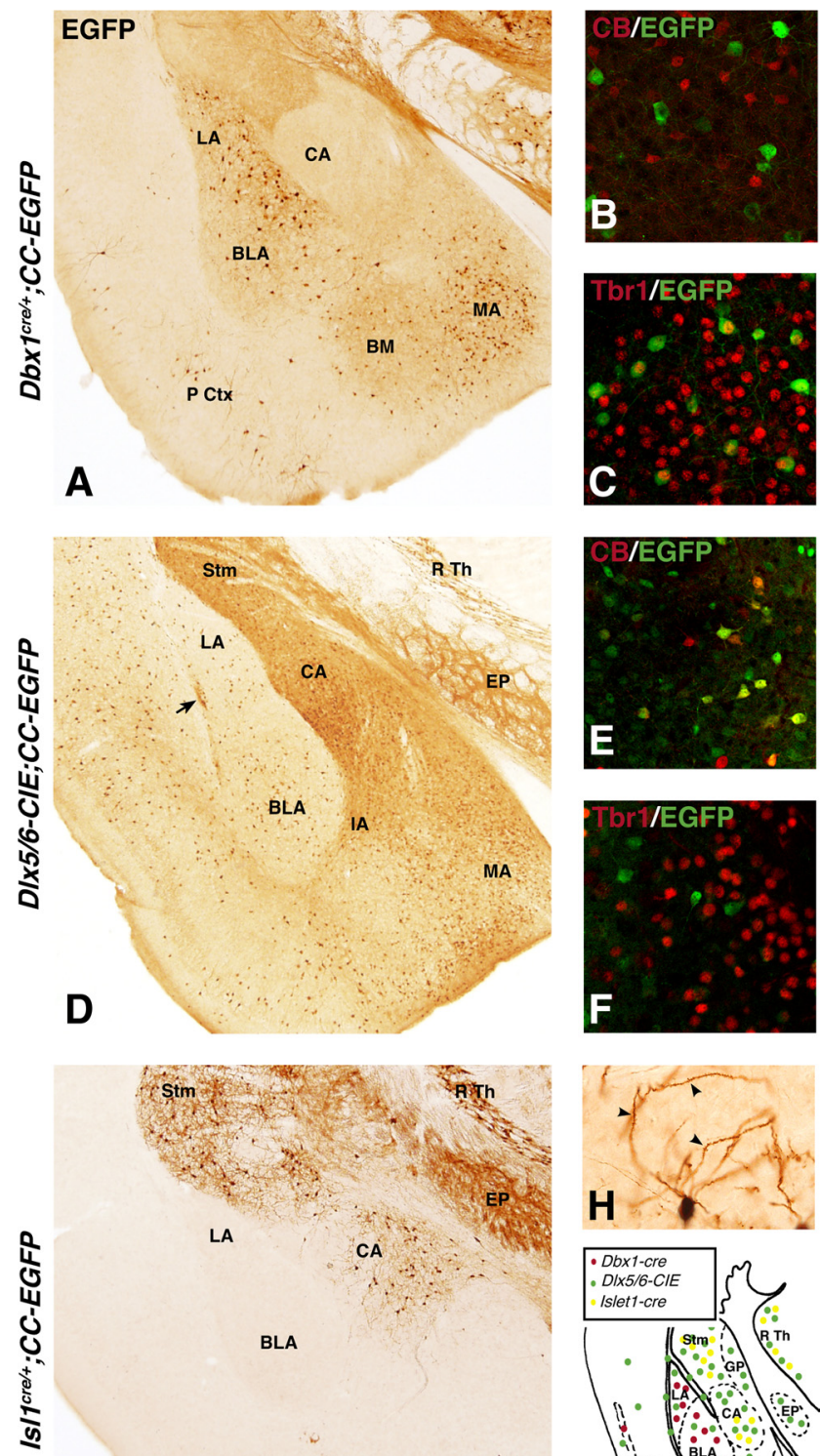

G
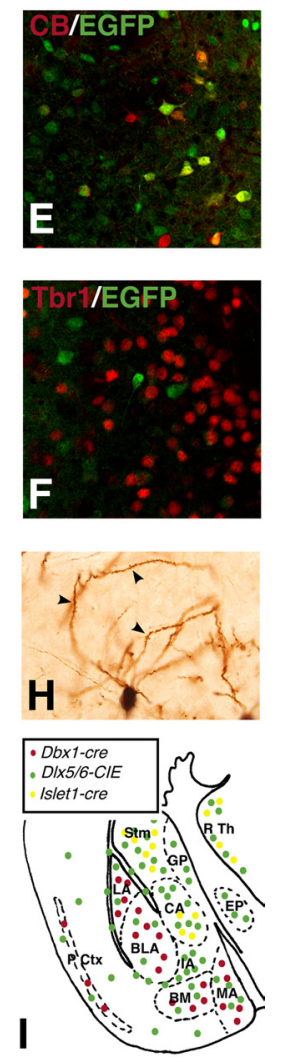

Figure 1. Fate-mapping analysis of amygdalar regions from mice expressing $D b \times 7^{7^{c r e} /+}$, $D / \times 5 / 6-C I E$, or $/ s / 1^{c r e /+}$ in combination with the CC-EGFP cre reporter mice. $A$, Double-transgenic adult animals for $D b x 1^{c r e /+}$ and CC-EGFP showed EGFP expression in many cells scattered in the lateral, basolateral, and medial amygdala, whereas only a few EGFP cells were detected in the basomedial and pyriform cortex regions. $\boldsymbol{B}, \boldsymbol{C}, D b \times 1^{\text {cre/+ }}$ fate-mapped cells in the basolateral complex did not express calbindin (B) but did express Tbr1 (C). D, Double-transgenic adult animals for D/x5/6-CIE and CC-EGFP showed intense EGFP staining in the central and medial amygdala and scattered EGFP cells in the basolateral complex. $\boldsymbol{E}, \boldsymbol{F}$, There was coexpression of calbindin in D/x5/6-CIE fate-mapped cells in the basolateral complex $(\boldsymbol{E})$, but there was no overlap with Tbr1 expression $(\boldsymbol{F})$. G, Double-transgenic adult animals for $/ \mathrm{s} / 1^{\mathrm{cre} /+}$ and C(-EGFP showed EGFP-positive cells restricted to the central amygdala. $\boldsymbol{H}$, High-power image reveals $|s| 1^{\text {cre/+ }}$ fate-mapped cells exhibit a medium-sized spiny striatal-like characteristic. $I$, Schematic diagram of fate-mapped cells from $D b x 1^{\text {cre/+ }}$ (red), DIX5/6-CIE (green), or $/ s / 1^{\text {cre/+ }}$ (yellow) at the level of the basolateral complex. Stm, Striatum; P Ctx, pyriform cortex; BM, basomedial amygdala; $R$ Th, reticular thalamus; EP, entopeduncular nucleus; GP, globus pallidus; MA, medial amygdala.

and BLA as well as throughout the cortex (Fig. 1D). Since Dlx5/6 progenitors essentially encompass the entire ventral telencephalon (including the MGE, LGE, and CGE), we have taken advantage of the Isl1 ${ }^{\text {cre/+ }}$ mice (Srinivas et al., 2001) to mark the contribution of vLGE progenitors to amygdalar neurogenesis. Our group previously suggested that the Isl1-positive vLGE gives rise to the striatal projection neurons (Stenman et al., 2003a). Indeed $\mathrm{Isl1}^{\text {cre/+}}$;CC-EGFP mice contained many recombined (i.e., fate-mapped) neurons in the striatum with a medium-sized, spiny appearance (Fig. 1G) (data not shown). In keeping with the fact that the CA possesses striatum-like characteristics (Swanson and Petrovich, 1998), Isll fate-mapped neurons were observed in the CA, but not in the LA/BLA (Fig. $1 G$ ). Because the EGFP filled the processes of the fate-mapped neurons, we found that many of these cells had a medium-sized spiny morphology (Fig. $1 \mathrm{H}$ ) characteristic of the GABAergic CA projection neurons (McDonald, 1982). Thus, the principal neurons of the CA appear to derive from Isl1positive vLGE progenitors (Fig. 1I), which also give rise to striatal projection neurons (Stenman et al., 2003a).

Unlike the Isl 1 fate maps, the $D b x 1$ and $D l \times 5 / 6$ fate maps showed many neurons scattered in multiple amygdalar nuclei (Fig. 1I). Interestingly, the EGFP cells in LA and BLA from the Dlx5/6 fate map appeared smaller than those from the $D b x 1$ fate map, suggesting that they give rise to interneuron subtypes, whereas the $D b \times 1$ derivatives may represent mainly projection neurons. To determine the neuronal subtypes labeled, we colocalized the EGFP staining with either Tbr1, which marks pallial projection neurons (Hevner et al., 2006) or the calcium binding proteins $\mathrm{CB}$ and calretinin $(\mathrm{CR})$, which mark interneuron subtypes in the amygdala (McDonald and Mascagni, 2001). Within the LA and BLA, $84 \%$ of the EGFP cells from the $D b x 1$ fate map also expressed Tbr1 (Fig. 1C) and few, if any, express $\mathrm{CB}$ (Fig. $1 B$ ). Conversely, the Dlx5/6 fate-mapped cells in the LA and BLA were never observed to express Tbr 1 (Fig. $1 F$ ), whereas $34 \%$ of the EGFP cells expressed CB (Fig. 1E). Although CR cells are fewer in the LA and BLA than the CB cells, none of these were fate mapped from the $D b x 1^{c^{c r e /+}}$, but some originate from the $D l \times 5 / 6$ expressing progenitors. Together, our fate-mapping experiments suggest the projection neurons in the LA and BLA derive, at least in part, from the $D b x 1$-expressing VP and the interneurons in and around these nuclei derive mostly from the Dlx5/6expressing ventral telencephalon (Fig. 1I).

We were intrigued by the unique placement of certain Dlx5/6derived EGFP cells found clustered adjacent to the LA and BLA on both the lateral and medial sides (Figs. $1 D$, arrows; $2 A, B$ ). They appeared to be paracapsular ITCs of the amygdala, which are interneurons that mediate signaling between the basolateral complex and the CA (for review, see Ehrlich et al., 2009). These cells were not observed in Isl 1 fate-mapped brains (Fig. 1G), suggesting that they derive from either the MGE or CGE or the dorsal LGE (dLGE). To better characterize these cells, we searched the literature for genes expressed in the LGE or MGE during development that label scattered cells within the amygdala. Previous studies showed that the winged helix/forkhead transcription factor Foxp2 was expressed in sporadic cells in the amygdalar region (Ferland et al., 2003). Indeed, these sporadic Foxp2 neurons appear to be ITCs that originate from the $D l \times 5 / 6$ expression domain (Fig. $2 C, D$ ). In addition, our group previously found that the homeobox protein Meis2 is expressed in the IA (Stenman et al., 2003b), which is the ventral extension of the paracapsular ITCs. Interestingly, Meis2 expression appears very similar to that of Foxp2 in the paracapsular and IA regions (Fig. 2 E). In fact, most Foxp2 cells in the paracapsular region and IA were also Meis2positive (Fig. $2 F$ ) (data not shown). The $\mu$ OR has previously been shown to mark at least a subpopulation of paracapsular ITCs as well as those in the IA (Jacobsen et al., 2006; Likhtik et al., 

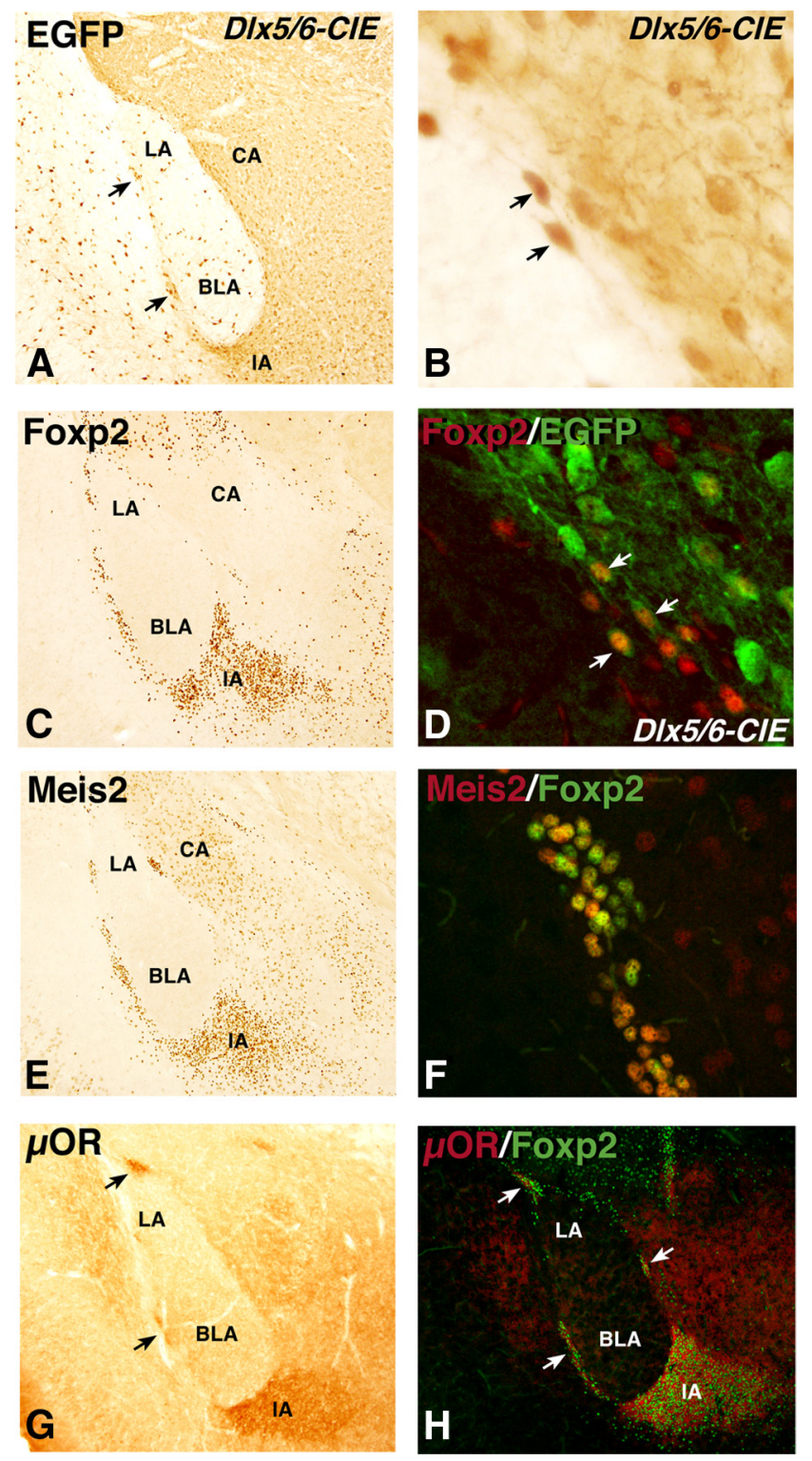

Figure 2. Characterization of the IA and paracapsular ITCs in the adult basolateral complex. $A$, Double-transgenic adult animals for DIx5/6-CIE and CC-EGFP showed robust EGFP expression in the IA. $\boldsymbol{A}, \boldsymbol{B}$, In addition, the D/x5/6-CIE fate map revealed EGFP expression in the ITCs located on the lateral side ( $\boldsymbol{A}$, arrows) and medial side ( $\boldsymbol{B}$, arrows). $\boldsymbol{C}, \boldsymbol{E}, \boldsymbol{G}$, The ITCs and the IA can be labeled by the expression of Foxp2 ( $(\boldsymbol{C})$, Meis2 (E), and $\mu 0 R$ (G). D, Many Foxp2-positive ITCs coexpress EGFP from the D/x5/6-CIE fate map. $F$, Nearly all Foxp2-positive paracapsular intercalated neurons also express Meis2. $\boldsymbol{H}$, Moreover, Foxp2-positive nuclei overlap extensively with the ITC marker $\mu O R$.

2008) (Fig. 2G). We found that Foxp2-stained nuclei overlapped specifically with $\mu \mathrm{OR}$ staining in these amygdalar regions (Fig. $2 H$ ).

\section{Amygdalar defects in Gsx2 mutants}

The homeobox gene, Gsx2 is expressed in LGE progenitors and the loss-of-function mutants display defects in cell types derived from the LGE, namely striatal projection neurons and olfactory bulb interneurons (Corbin et al., 2000; Toresson et al., 2000; Toresson and Campbell 2001; Yun et al., 2001, 2003; Waclaw et al., 2004, 2006). However, the amygdalar complex has not been carefully analyzed in Gsx2 mutant embryos. To study the role of Gsx2 in the developing amygdala, we used an EGFP knock-in to the Gsx2 locus that allows for a short-term fate map of Gsx2- expressing cells (Wang et al., 2009). At late gestation stages (E16.5 and E18.5), Gsx2 is highly expressed in the dLGE (Yun et al., 2001; Waclaw et al., 2009), and Gsx2 $2^{E G F P /+}$ embryos show high levels of EGFP in the dLGE (Wang et al., 2009) (Fig. 3). Interestingly, at E18.5, EGFP-positive cells that are continuous with the dLGE region are observed in the lateral migratory stream and around the developing basolateral complex (Fig. $3 A-C$ ). A number of the EGFP-positive cells in the lateral migratory stream appear to express Foxp2 (Fig. 3B), as is the case with some of EGFP-positive cell clumps in the paracapsular region of the basolateral complex (Fig. 3C). As would be predicted, the Gs $x 2^{E G F P}$-expressing cells are mostly distinct from, but complementary with, cells that express the pallial differentiation marker Tbr1 (Fig. 3G-I). These findings suggest a dLGE origin of the ITCs. It should be noted, however, that EGFP expression from the Gsx2 locus only represents a short-term fate map of Gsx2-expressing cells, and it is likely that the EGFP is downregulating in the amygdalar region already by this stage.

By crossing the recombined (i.e., null) Gs $x 2$ allele $\left(G s \times 2^{R A}\right)$ (Waclaw et al., 2009) with Gs $x 2^{E G F P /+}$ mice, we followed the fate of Gsx2-null cells in the lateral migratory stream and developing amygdala. In stark contrast to the Gs $x 2^{E G F P /+}$ embryos, Gs $x 2^{E G F P / R A}$ embryos lack Foxp2 and thus Foxp2/EGFP colocalization in the lateral migratory stream and paracapsular region (Fig. 3D-F). In fact, the striatal complex in Gs $x 2^{E G F P / R A}$ embryos, as marked by Foxp2, is severely reduced in size (Fig. 3D). Remarkably, $\sim 76 \%$ of the EGFP cells in the lateral migratory stream of Gs $x 2^{E G F P / R A}$ embryos were observed to express Tbr1 ectopically (Fig. $3 J-L$ ) compared with only $\sim 5 \%$ in the Gs $x 2^{E G F P /+}$ embryos (Fig. $3 G-I)(n=3 ; p<0.001)$. These findings suggest that the dLGE cells in the lateral migratory stream and amygdala have changed fate toward pallial identity (i.e., Tbrl expression). This is in line with previous studies that have shown that Tbr1 is expanded in the ventrolateral telencephalon of Gs $x 2$ mutants (Yun et al., 2001) and that Gsx2 mutants have reductions in cells that emanate from the pallio-subpallial boundary and migrate toward the developing amygdala (Stenman et al., 2003c; Carney et al., 2006, 2009). Thus, Gsx2 mutants seem to have reduced amygdalar contributions from the dLGE and a concomitant increase in neurons derived from the VP.

Postnatal analysis of the amygdalar complex in Gsx2 mutants has not been possible because of embryonic lethality at birth (Szucsik et al., 1997). To circumvent this problem, we created a conditional mutant allele $\left(G s \times 2^{f l}\right)$ that contains loxP sites flanking exon 2, which includes the entire homeodomain (Waclaw et al., 2009). To generate a telencephalon-specific loss of function for Gsx2, we first attempted to use the Foxg1 ${ }^{\text {cre }}$ mice (Hébert and McConnell, 2000); however, this cre line resulted in widespread recombination of the $G s \times 2^{f l}$ allele and perinatal lethality (data not shown). This was not entirely unexpected as these mice were originally found to induce extensive nontelencephalic recombination on certain outbred backgrounds (Hébert and McConnell, 2000). To overcome the lethality issue, we have used Foxg $1^{t T A /+}$ mice, which express the tetracycline transactivator $(t T A)$ gene specifically in the telencephalon (Hanashima et al., 2002; Waclaw et al., 2009), in combination with tet-O-cre mice (Perl et al., 2002). The breeding strategy to generate a telencephalon-specific Gs $x 2$ mutant is depicted in Figure 4A. Because this is a "Tet-off" system, the presence of the $t T A$ and cre alleles is sufficient to drive recombination within the Foxg1 expression domain. Gsx2 conditional mutants (i.e., Foxg $1^{\text {tTA/+ }}$; tet-O-cre; Gs $x 2^{\text {fl/fl }}$ ) show a nearly complete loss of Gsx2 in the LGE at E12.5 (Fig. 4C) compared 


\section{GsX2EGF/+}
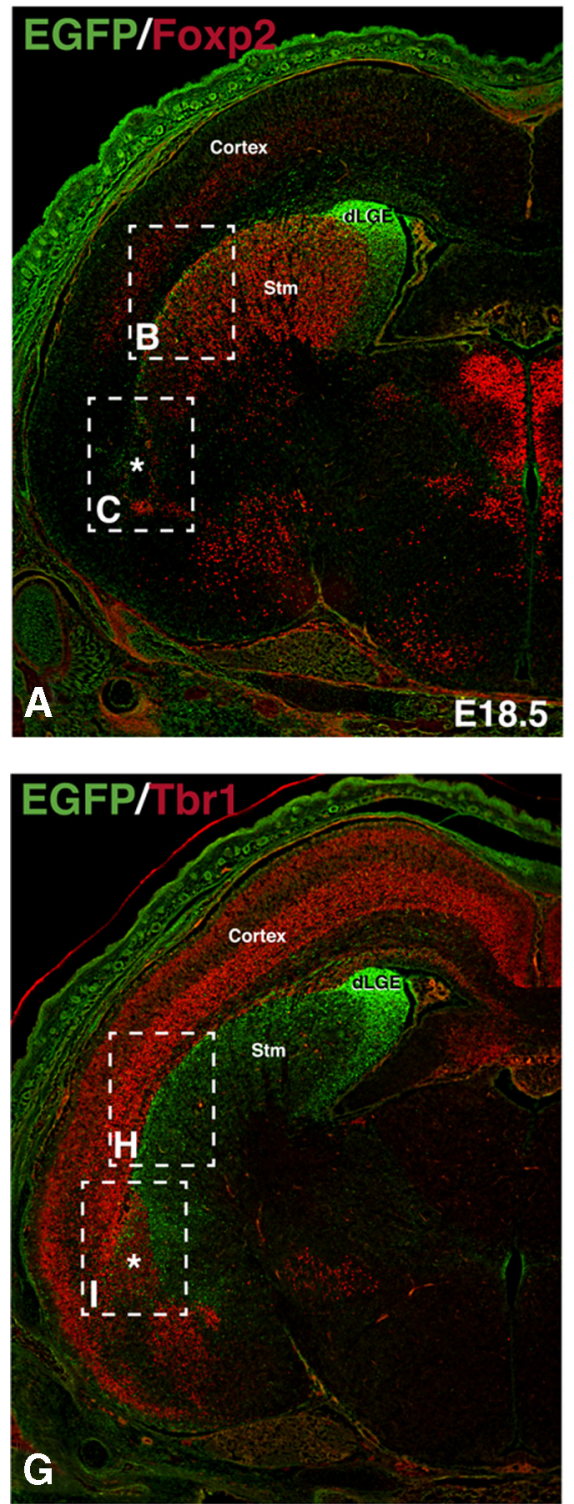

B
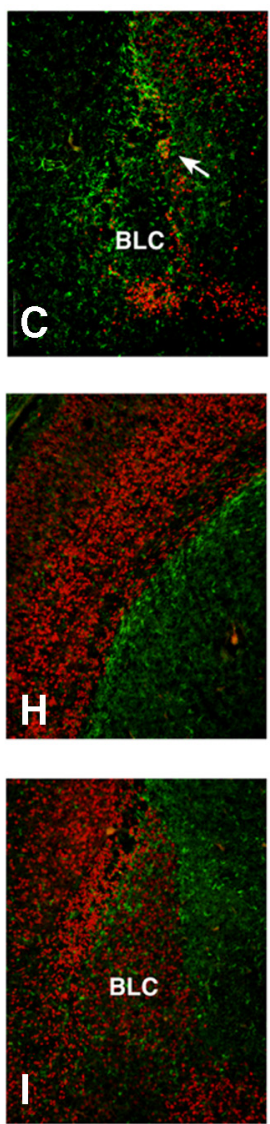

\section{GSX2EGFP/RA}
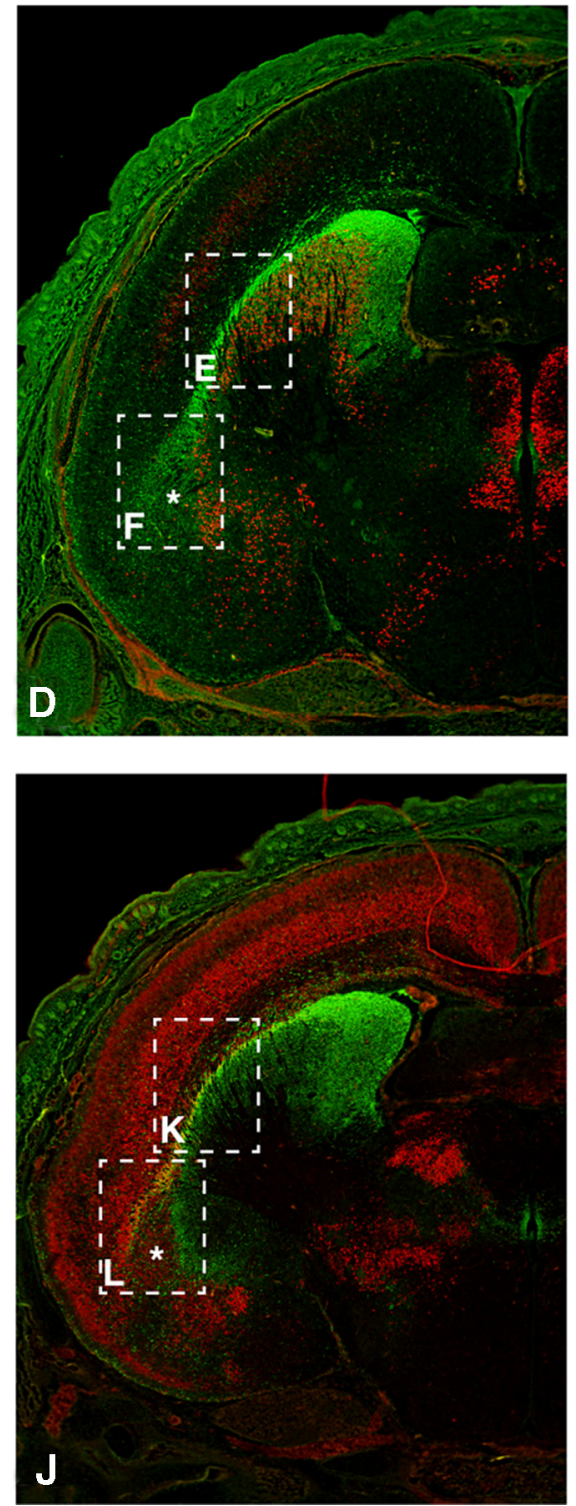
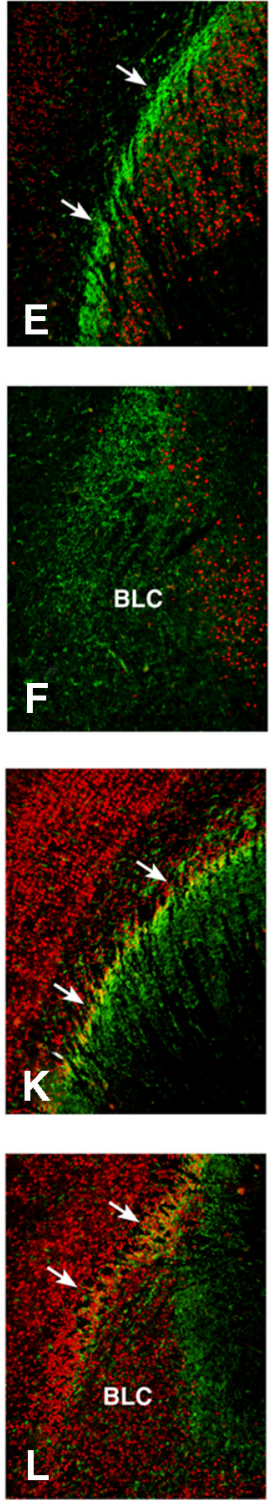

Figure 3. Characterization of GSX2 mutant cells in the dLGE, lateral migratory stream, and forming basolateral complex. A, At E18.5, GsX2 ${ }^{\text {EGPP/+ }}$ embryos express high levels of EGFP in the dLGE. $\boldsymbol{A}-\boldsymbol{C}$, In addition, a lateral stream of EGFP-positive cells is observed in the ventral lateral telencephalon at the dLGE $(\boldsymbol{A})$, dorsal border of the striatum $(\boldsymbol{B})$, and developing amygdala around the basolateral complex (C). Foxp2-positive cells are also observed at dorsal-most border of the striatum (B) and surrounding the forming basolateral complex (C). The arrows in $($ point to EGFP/Foxp2 double-positive cells around the basolateral complex. $\boldsymbol{D}, \boldsymbol{E}$, GSX2 mutants (GSX2 ${ }^{E G F P / R A}$ ) exhibit a dense lateral stream of EGFP-positive cells at the dorsal border of the striatum (D; $\boldsymbol{E}$, arrows) that is not Foxp2-positive (E).F, In addition, there are no Foxp2/EGFP double-positive cells in the developing basolateral complex of Gsx2 mutants. G-I, At E18.5, Tbr1/EGFP double staining in Gsx2 $2^{E G F /+}$ embryos reveals complimentary dorsal (Tbr1) and ventral (EGFP from Gsx2) domains in the lateral migratory stream $(\boldsymbol{H})$ and developing basolateral amygdalar complex (I). $J-L$, GSX2 mutants show a significant overlap of Tbr1 and EGFP staining in the lateral migratory stream $(\boldsymbol{J}, \boldsymbol{K})$ and developing basolateral complex $(\boldsymbol{J}, \boldsymbol{L})$. BLC, Basolateral complex; Stm, striatum.

with controls (Fig. 4B), with only a few Gsx2-positive cells detectable in the LGE at this early stage (Fig. 4C). By E18.5, no Gsx2-positive cells were detected in the conditional mutant telencephalon (Fig. 4, compare E, D). Gsx2 conditional mutants exhibited nearly identical phenotypes in the embryonic striatum and olfactory bulb (data not shown) with those previously published in germ-line Gsx2 mutants (Corbin et al., 2000; Toresson et al., 2000; Yun et al., 2001).

Gsx2 conditional mutants were viable to adult stages and showed a severe reduction in striatal volume (data not shown) as well as clear abnormalities in the morphology of the amygdalar complex (Fig. 5). To address the general morphology of the basolateral complex, we analyzed Tbr1 expression, which labels the projection neurons in both the LA and BLA (Fig. 5A). Gsx2 conditional mutants display an expanded Tbr 1 expression domain in the LA, but relatively normal expression in the BLA (Fig. 5, compare outlined regions in $B$ with $A$ ). Interestingly, very few specific markers of the LA have been identified. The MADS box transcription factor Mef2c was previously shown to be expressed in the embryonic amygdalar region (Lyons et al., 1995). We have further analyzed Mef2c gene and protein expression and found that it is highly enriched in the LA compared with the BLA (Fig. $5 C$ ) (data not shown). Moreover, Mef2c expression is expanded in the Gsx2 conditional mutant LA (Fig. 5D) similar to the expansion of Tbr 1 in the mutant LA (Fig. 5B). Indeed, Gs $x 2$ conditional mutants showed a $55 \%$ increase in the area of the Mef2c expres- 

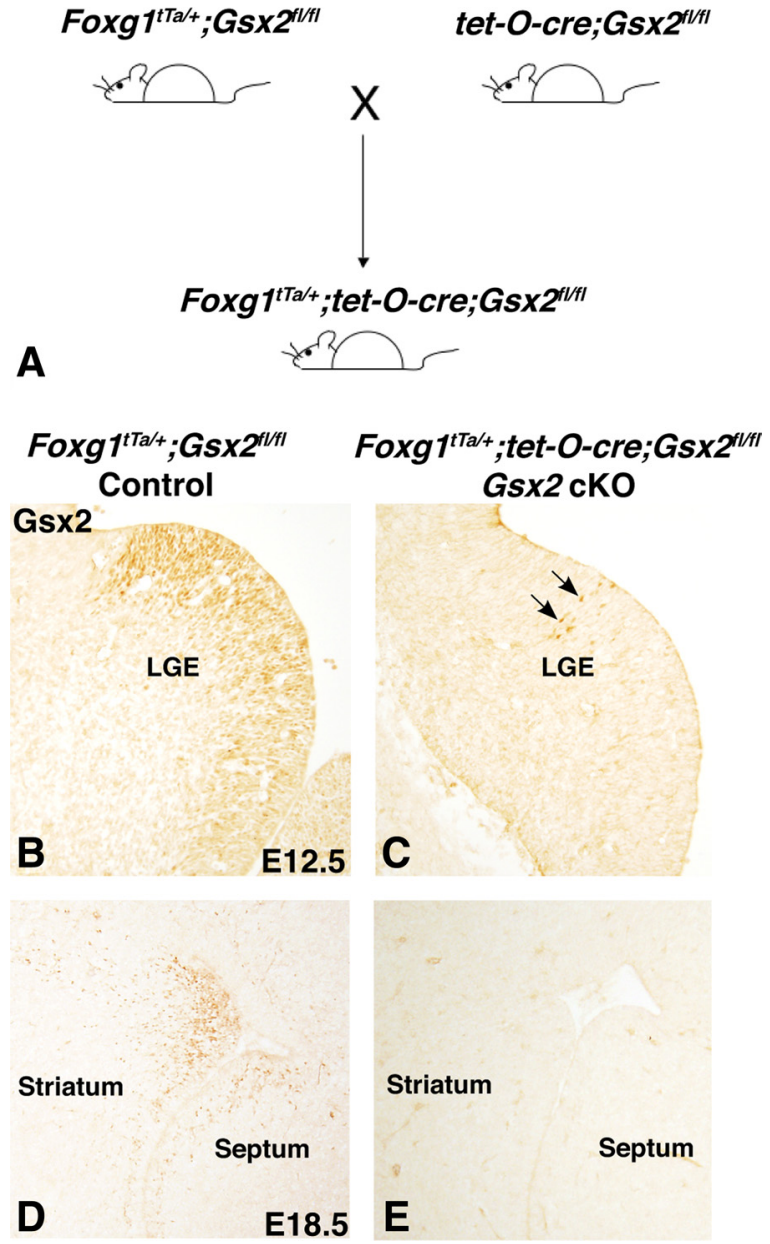

Figure 4. Breeding strategy and Gsx2 expression in telencephalon-specific Gsx2 conditional

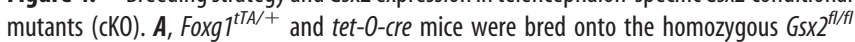
background and then bred together to generate triple-transgenic telencephalon-specific Gsx2 conditional mutants $\left(F o x g 7^{t T A /+} ;\right.$ tet-0-cre;GsX2 $\left.2^{f / f f}\right) . B, C$, At E12.5, very few Gsx2-positive cells can be detected in the LGE of conditional mutants ( $\boldsymbol{C}$, arrows) compared with controls (B). $\boldsymbol{D}, \boldsymbol{E}$, By E18.5, Gsx2 expression (D) is not detected in the conditional mutant telencephalon $(\boldsymbol{E})$.

sion domain compared with controls $(n=3 ; p<0.001)$. However, the density of Mef2c cells in the Gsx2 conditional mutant and controls was not different $(3996 \pm 148$ and $4474 \pm 362$ cells $/ \mathrm{mm}^{2}$, respectively; $n=3 ; p=0.15$ ). Thus, more Mef2c cells are present in the LA of Gsx2 conditional mutants. We further addressed the BLA complex by analyzing the expression of the ETS transcription factor Er81, which is found in the BLA and not the LA (Stenman et al., 2003b) (Fig. 5E). Gsx2 conditional mutants show a similar area of Er81 expression in the BLA (Fig. $5 F$ ) compared with controls (Fig. $5 E$ ) (104\% of control; $n=3 ; p=$ $0.34)$. Moreover, the density of Er81 cells in the BLA is not different between Gsx2 conditional mutants and controls (1036 \pm 88 and $1018 \pm 76$ cells $/ \mathrm{mm}^{2}$, respectively; $n=3 ; p=0.44$ ). No markers that are specific to the CA have been identified thus far; however, we found that the serotonin receptor Htrld is highly expressed in this nucleus (Fig. 5G). Although the CA appears to derive from the vLGE and shares striatal characteristics, the Htrld expression domain in the CA of Gsx2 mutants was nearly identical (i.e., $98 \%)$ with controls (Fig. $5 H)(n=3 ; p=0.43)$. This is despite the fact that the striatum in the germ-line Gsx2 mutant is reduced in size by $>50 \%$ (Toresson and Campbell, 2001). Together, our results suggest that the LA is specifically affected in Gsx2 conditional mutants.

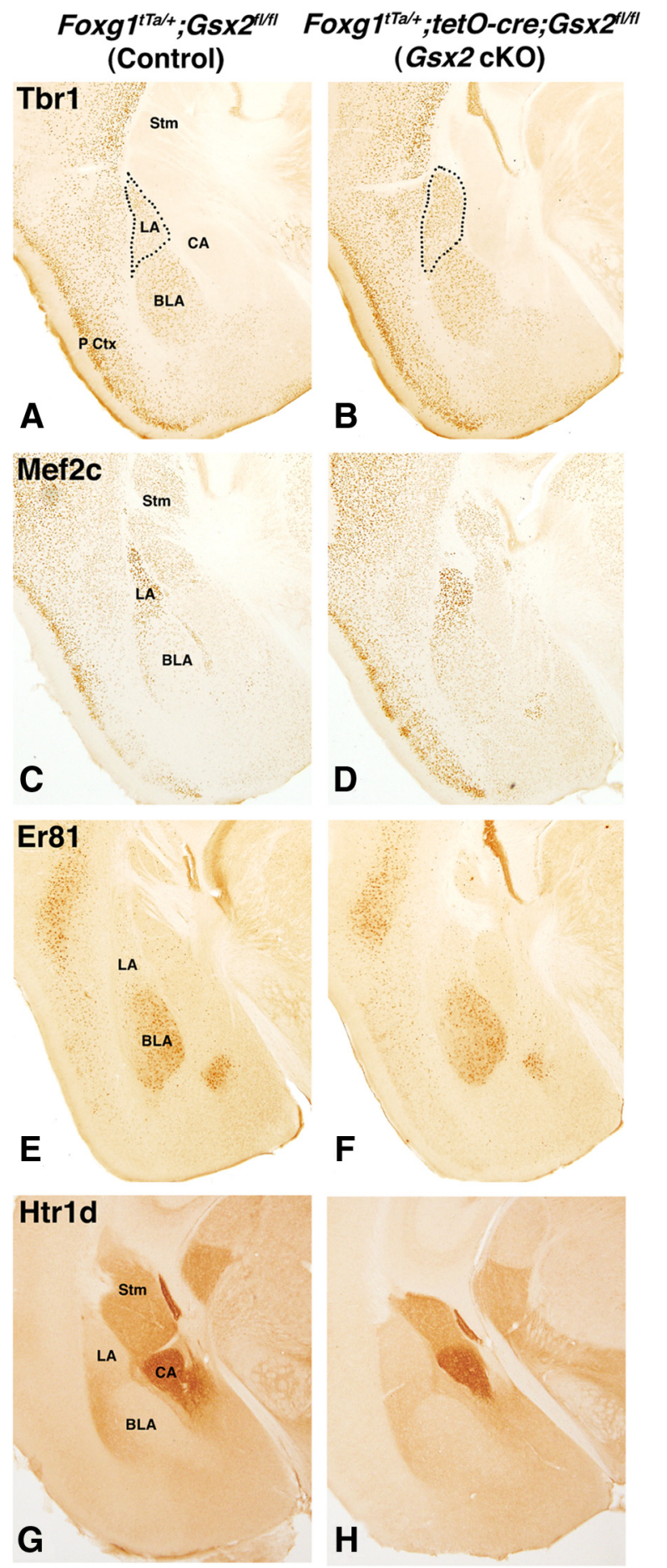

Figure 5. Characterization of amygdalar defects in postnatal telencephalon-specific G5x2 conditional mutants (CKO). $\boldsymbol{A}$, At adult stages, Tbr1 expression labels the lateral and basolateral amygdala. The lateral amygdala is outlined in dashed lines. Gs 2 conditional mutants show expanded Tbr1 expression in the anterior region of the lateral amygdala (outlined in dashed lines in $\boldsymbol{B}$ ). $\boldsymbol{C}$, Mef2c expression is enriched in the lateral amygdala in the adult. $D$, Gs 2 conditional mutants show an expanded anterior domain of Mef2 2 in the lateral amygdala. $\boldsymbol{E}$, The basolateral amygdala is marked by Er81 expression in controls. $F$, No obvious changes in the Er81 expression domain were observed in the basolateral amygdala of Gsx2 conditional mutants. $\boldsymbol{G}, \boldsymbol{H}$, The serotonin receptor Htr1d expression in the central amygdala was similar in controls $(\boldsymbol{G})$ and $G s \times 2$ conditional mutants $(\boldsymbol{H})$. Stm, Striatum; $\mathrm{P}$ Ctx, pyriform cortex.

Previous studies have shown the VP, as marked by Dbx1, is expanded in the Gsx2 mutant LGE (Yun et al., 2001; Stenman et al., 2003c; Carney et al., 2009). To determine the embryological origin of the LA phenotype in Gsx2 conditional mutants, we used 

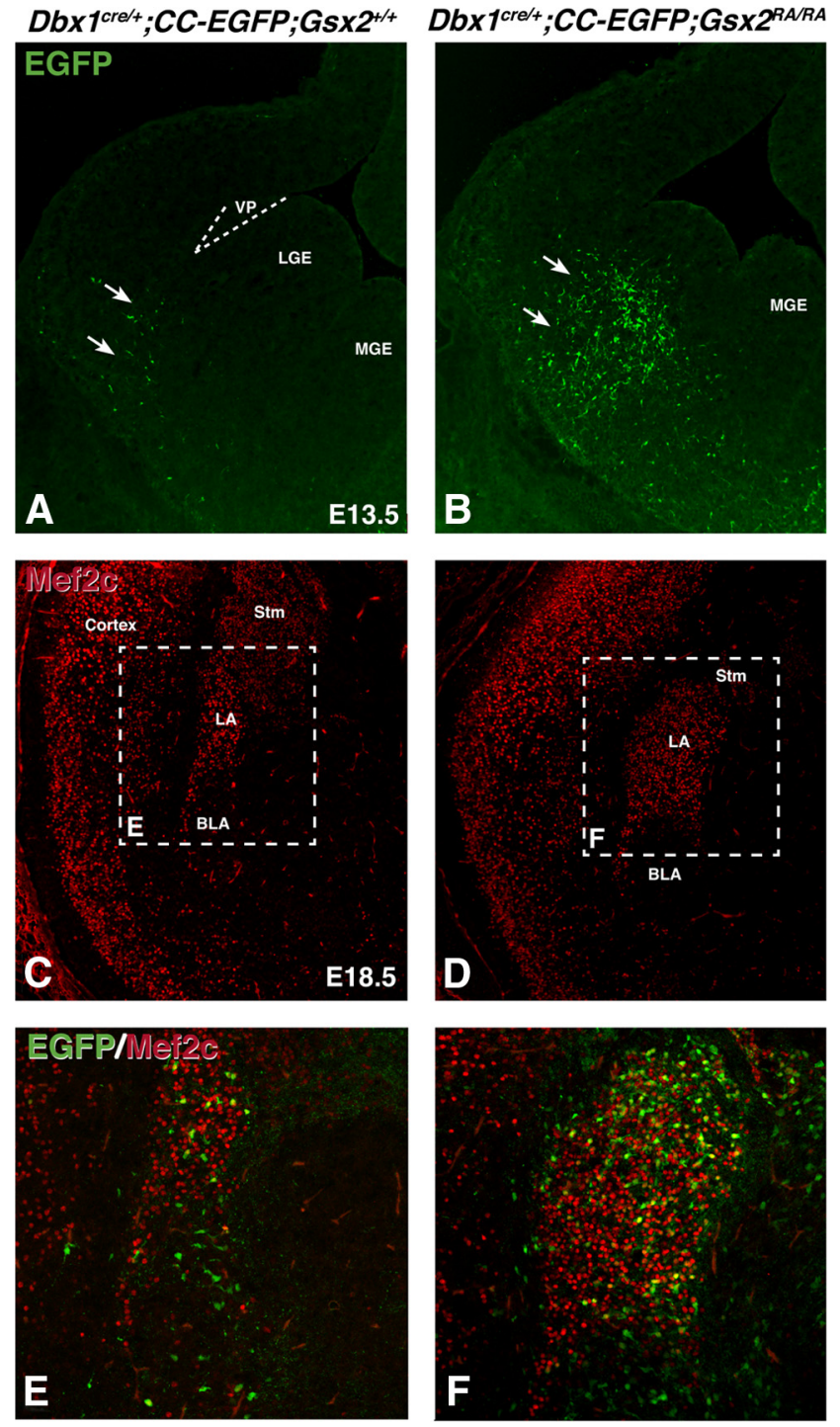

Figure 6. Fate mapping the $D b \times 1$ domain of the ventral pallium on the Gsx2 mutant background. At E13.5, a small number of $D b \times 1$ fate-mapped cells are observed migrating from the ventral pallium (marked by dashed lines) toward the ventral lateral telencephalon in control embryos ( $\boldsymbol{A}$, arrows). At this stage, there is a large increase in EGFP-positive cells throughout the GsX2 mutant LGE ( $\boldsymbol{B}$, arrows). $\boldsymbol{C}$, At E18.5, Mef2c expression labels the lateral amygdala and outlines the basolateral amygdala in control embryos. D, GsX2 mutant embryos show an expanded Mef2c expression domain in the lateral amygdala. $\boldsymbol{E}$, Dbx 1 fate-mapped cells (EGFPpositive) in the lateral amygdala express Mef2c in control embryos at E18.5. F, Many of the increased Mef2c-positive cells in the lateral amygdala of GsX2 mutants are also Dbx1 fate mapped (EGFP-positive) $(\boldsymbol{F})$. Stm, Striatum.

a fate map of the VP using $D b \times 1^{\text {cre/+ }}$ mice and recombination reporter CC-EGFP mice on the germ-line Gsx2 mutant background (i.e., $G s \times 2^{R A / R A}$ ). As predicted, at E13.5, there is a large increase in $D b x 1$ fate map cells in the ventrolateral telencephalon of the Gsx2 mutant LGE (Fig. 6B) compared with controls, which only expressed EGFP in progeny of the VP streaming toward the developing amygdala (Fig. 6A). This approach is extremely informative because it allows for us to follow the expanded $D b \times 1$ population at least until E18.5 in the Gsx2 mutant amygdala. Indeed, at E18.5, germ-line Gsx2 mutants show a dramatic expansion of the LA as marked by Mef2c expression (Fig. 6, compare $D, C$ ). Accordingly, many more EGFP-positive cells (i.e., Dbx1 fatemapped cells) are observed in the LA of Gs $x 2$ mutants (Fig. $6 F$ ) $(170 \pm 14$ cells; $n=3)$ compared with controls (Fig. $6 E)(33 \pm 4$ cells; $n=3$ ). This represents a nearly sixfold increase in Dbx1derived cells within the LA of Gsx2 mutants; however, we only observed approximately a twofold increase in the BLA of these mutant ( $46 \pm 4$ cells compared with $21 \pm 1$ cells in controls; $n=$ 3). In control embryos, the vast majority (i.e., $81 \%$ ) of the $D b x 1$ fate-mapped cells in the LA are Mef2c-positive. Moreover, most (i.e., $83 \%$ ) of the EGFP-positive cells in the mutant LA are also Mef2c-positive (Fig. 6F). The increase in the Dbx1-derived cells of the E18.5 mutant LA, compared with the BLA, is consistent with the rather selective increase in cell numbers observed in the LA versus BLA of adult Gs $x 2$ conditional mutants. Thus, the Dbx1 fate-mapping experiments suggest that the LA phenotype in Gs $x 2$ mutants is a consequence of the expansion of the VP (i.e., $D b x 1$ cells) in the LGE.

Short-term fate mapping using the $G s \times 2^{E G F P}$ allele in control and Gs $x 2$ mutant embryos revealed that at least some of the ITCs in the paracapsular and IA regions of the amygdala derive from the LGE and in particular the dLGE (Fig. 3). We then analyzed the adult telencephalon of the Gsx2 conditional mutants to determine whether ITCs are generated in a normal manner. Both Foxp2 and Meis 2 expression were reduced in the paracapsular and IA regions of the Gsx2 conditional mutant amygdala (Fig. $7 B, E$ ) compared with controls (Fig. $7 A, D$ ). Indeed, the area of Foxp 2 expression in the IA was reduced by $61 \%$ in the Gs $x 2$ conditional mutant compared with controls $(n=3 ; p<0.01)$. At more caudal levels, the IA is not apparent but paracapsular ITCs (as marked by Foxp2) are present on the lateral and medial sides of the basolateral complex (Fig. $7 G$ ). As is the case at more rostral levels, Gsx2 conditional mutants show fewer Foxp2 ITCs in the paracapsular regions (Fig. $7 H$ ). In line with these findings, $\mu \mathrm{OR}$ is also reduced in the paracapsular and IA regions of Gs $x 2$ conditional mutants (Fig. $7 K$ ) compared with controls (Fig. 7J). These results support the notion that ITCs derive from the LGE and require Gsx2 for their normal generation.

\section{Amygdalar defects in Sp8 mutants}

To further address the origin of the ITCs, we have examined the amygdala of $S p 8$ conditional mutants. $S p 8$ is a zinc-finger transcription factor, which is expressed in the dLGE and requires Gsx2 for its normal expression in this region (Waclaw et al., 2006). In addition, we showed that conditional deletion of Sp8 using Dlx5/6-CIE (i.e., throughout the ventral telencephalon) results in defects in the normal development of the dLGE as well as the generation of specific olfactory bulb interneuron populations, namely the CR neurons. Despite this, conditional deletion of $S p 8$ in the ventral telencephalon does not result in abnormal dorsal-ventral patterning of the LGE (Waclaw et al., 2006). Moreover, the size of the basolateral complex, as marked by Tbr 1 expression, was not different between $S p 8$ conditional mutants and controls ( $94 \%$ of control; $n=3 ; p=0.31$ ). Nevertheless, $S p 8$ conditional mutants have similar reductions in the ITCs compared with Gsx2 conditional mutants. Foxp2-, Meis2-, and $\mu \mathrm{OR}-$ positive cells are barely detectable in the paracapsular regions of Sp8 conditional mutants (Fig. 7C,F,I,L). In addition, the area of Foxp2 expression in the mutant IA is reduced in size by $59 \%$ compared with controls (Fig. 7, compare $C, F$, with $A, D)(n=3$; $p<0.01$ ), which is similar to that of the Gs 2 conditional mutants (Fig. 7 B,E). These data suggest that $G s x 2$ and $S p 8$ in the dLGE are necessary for normal Foxp2, Meis2, and $\mu \mathrm{OR}$ expression in the ITCs of paracapsular region and the IA. Moreover, they strongly suggest that a large portion of the ITCs originates in the dLGE. 


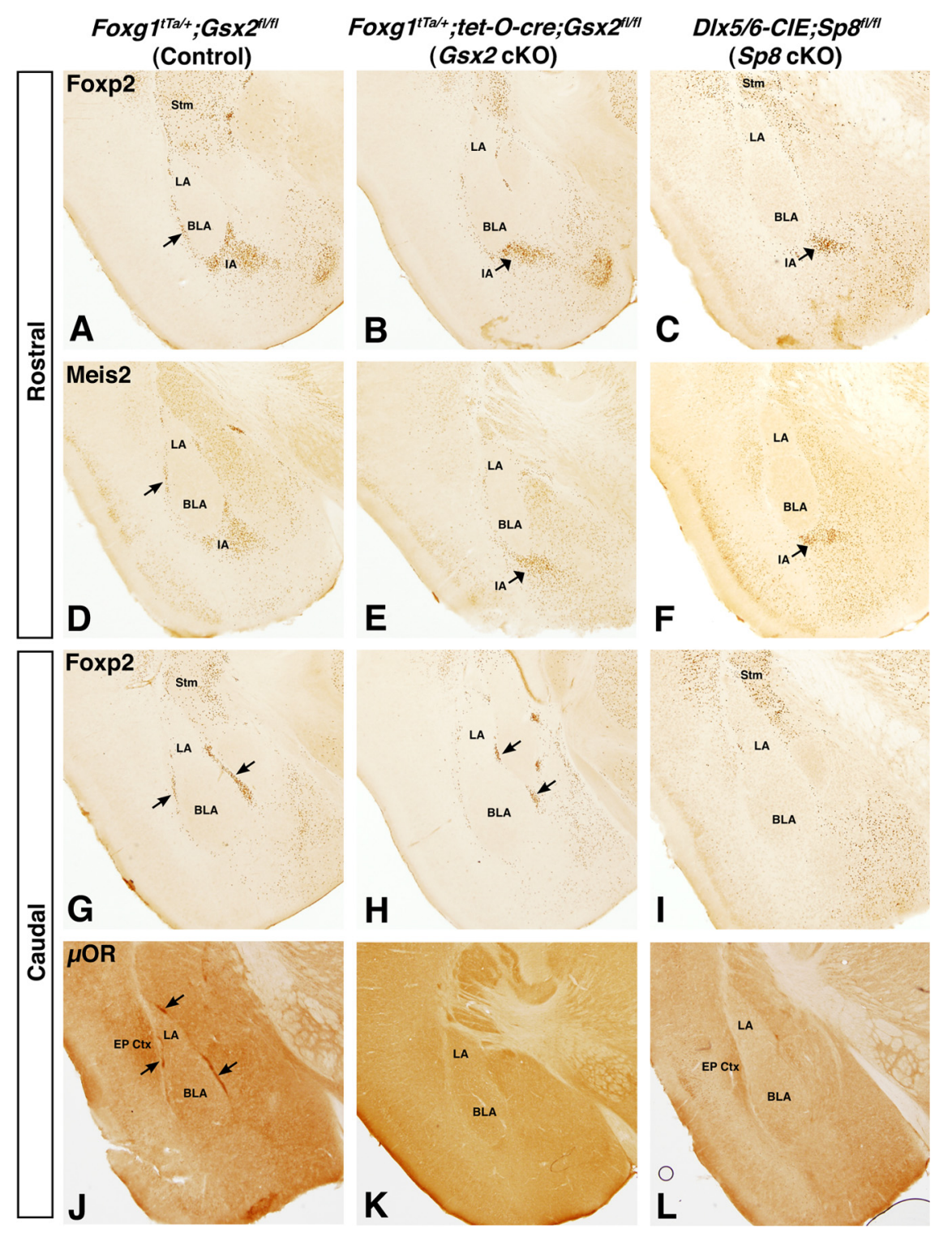

Figure 7. Genetic evidence the dLGE contributes to the IA and paracapsular ITCs in the adult basolateral complex. Foxp2 and Meis2 expression mark the IA and paracapsular ITCs on the lateral and medial regions of the basolateral complex $(\boldsymbol{A}, \boldsymbol{D}, \mathbf{G}$, arrows) in control brains. The expression domain of Foxp2 and Meis 2 in the IA is noticeably reduced in $G s \times 2$ CKO ( $\boldsymbol{B}, \boldsymbol{E}$, arrows) and Sp8 cKO $(\boldsymbol{C}, \boldsymbol{F}$, arrows) brain sections. In addition, fewer Foxp2 and Meis2 ITCs are observed around the GsX2 CKO $(\boldsymbol{B}, \boldsymbol{E}, \boldsymbol{H})$ and Sp8 cKO $(\boldsymbol{C}, \boldsymbol{F}, \boldsymbol{I})$ basolateral complex. However, it should be noted that ITCs on the medial side of the basolateral complex in GsX2 cK0 were observed at caudal levels (H, arrows). $\mu$ OR staining also marks the ITCs around the basolateral complex (J, arrows). Almost no $\mu 0 R$ staining is observed in GsX2 $\mathrm{CKO}(\boldsymbol{K})$ and Sp8 CKO $(\boldsymbol{L})$ adult brain sections. Note that $\mu 0 \mathrm{R}$ staining in the endopyriform cortex (EP $(\mathrm{Ctx})$ can be observed in control $(\boldsymbol{J})$, Gsx2 cKO $(\boldsymbol{K})$, and Sp8 cKO ( $\boldsymbol{L})$ brain sections. Stm, Striatum.

\section{Discussion}

Comparing the fate maps of Dbxl-, Dlx5/6-, and Isl1-expressing cells together with Gsx2 and Sp8 mutant analysis has allowed us to develop a model of the developmental origins of neuronal subtypes involved in the amygdalar fear circuit (Fig. 8). Our results demonstrate that the projection neurons of the LA and BLA arise from pallial progenitors, whereas those in the CA originate in the subpallium. Furthermore, it appears that the interneurons that populate this circuit also arise in the subpallium. In particular, the ITCs appear to derive from the dLGE, whereas the interneurons in the LA and BLA likely share origins with the cortical interneurons in the MGE and/or CGE.

The LA and BLA comprise the input to the amygdalar fear circuit (Paré et al., 2004; Ehrlich et al., 2009), and thus a pallial origin has been previously suggested (Swanson and Petrovich, 1998). The specific pallial progenitor domain(s) that contribute to the projection neurons in these amygdalar nuclei remains unclear. Previous studies had suggested that the LA and BLA were populated by projection neurons derived from the VP and lateral pallium, respectively (Medina et al., 2004). The fate map results using $D b \times 1$-cre mice (Bielle et al., 2005), as well as those published by Hirata et al. (2009), show that the $D b x 1$-expressing VP gives rise to projection neurons of both the LA and BLA. Accordingly, our previous findings showed that loss of VP identity in the Tlx mutant mice correlates with severe reductions in both the LA and BLA (Stenman et al., 2003b).

No amygdalar defects have been reported in Gsx2 mutants; however, these animals are not postnatally viable (Szucsik et al., 1997), and the full organization of the amygdala is difficult to examine at embryonic stages. Using a conditional inactivation strategy, we have obtained viable Gsx2 mutants and found that the LA, as marked by Tbr 1 and Mef2c, is specifically enlarged, whereas the BLA is not altered. Previous studies have shown that markers of the VP are expanded in Gsx2 mutants, suggesting an increase in this progenitor domain (Yun et al., 2001; Stenman et al., 2003c; Carney et al., 2009). Indeed, we show here that Gsx2 mutants display an increase in Dbx1-derived cells from the VP within the ventrolateral telencephalon. Moreover, we observed a nearly sixfold increase in $D b \times 1$-derived cells in the forming LA compared with slightly more than a twofold increase in the BLA. The same proportion of cells in the mutant LA expressed Mef2c as was observed in the control, suggesting that Gsx2 mutants have a selective expansion of LA progenitors within the VP. Thus, there may be a dorsoventral topography of progenitors within the VP, with the LA progenitors directly adjacent to the palliosubpallial boundary. BLA progenitors, however, may reside adjacent to the lateral pallium, which has also been suggested to give rise to the BLA (Medina et al., 2004).

The CA represents the output of the amygdalar fear circuit (Paré et al., 2004; Ehrlich et al., 2009) and exhibits many characteristics of the striatum including medium-sized spiny projection neurons (McDonald, 1982). These similarities have led to the speculation that the CA and striatum may share a common origin (Swanson and Petrovich, 1998). The striatum derives from the vLGE, which contains Isl1-expressing cells in the subventricular zone and in maturing striatal projection neurons (Stenman et al., 2003a). Using the Isl1-cre mice (Srinivas et al., 2001), we observed fate-mapped neurons specifically within the CA. Moreover, these neurons were medium-sized with spiny dendrites suggesting that they were CA projection neurons (McDonald, 


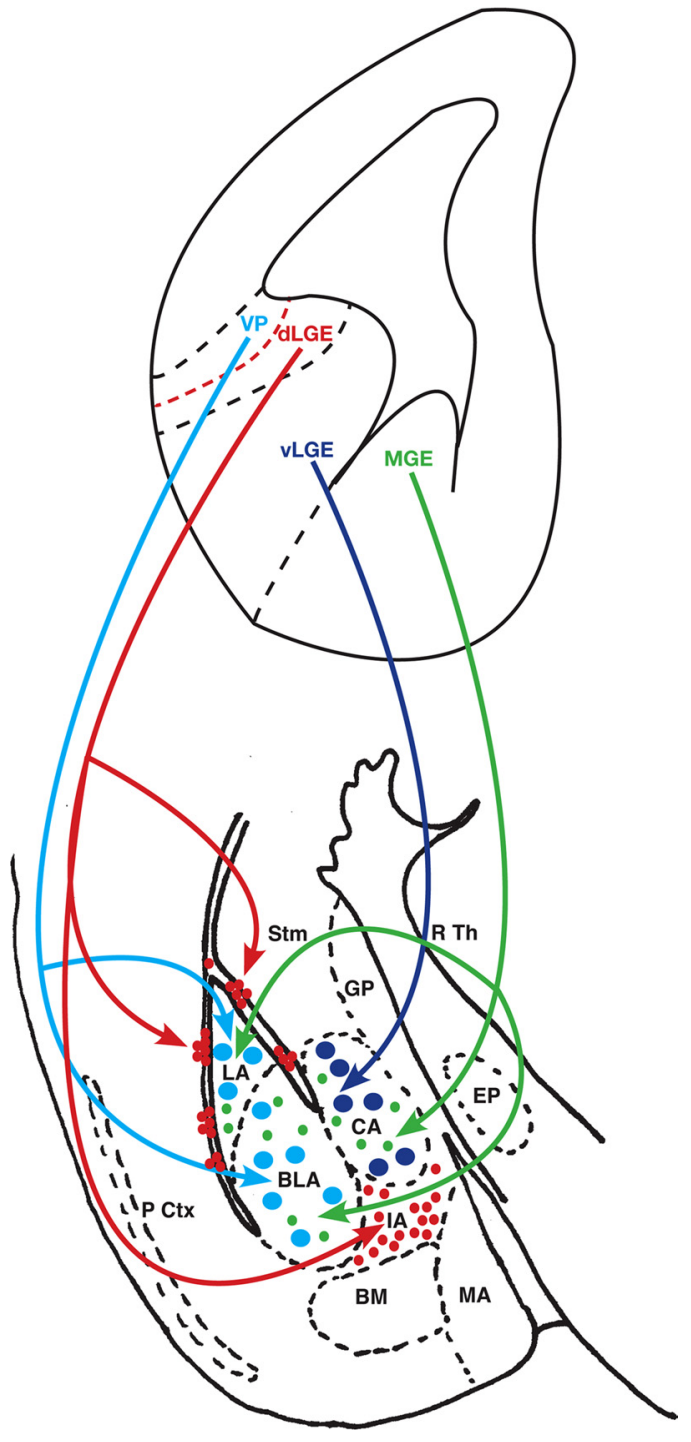

Figure 8. Schematic of lateral telencephalic progenitors domains contributing to the neuronal constituents of the amygdalar fear circuit. The VP (in blue) gives rise to projection neurons in the LA and BLA. The dLGE (in red) is a major source of the paracapsular ITCs and IA of the amygdala. The vLGE (in purple) gives rise to projection neurons in the CA. The MGE (in green) and likely CGE generate interneurons that reside in the LA, BLA, and CA. Stm, Striatum; P Ctx, pyriform cortex; BM, basomedial amygdala; $R$ Th, reticular thalamus; $E P$, entopeduncular nucleus; GP, globus pallidus; MA, medial amygdala.

1982). The CA can be divided into two distinct portions, a medial $(\mathrm{CAm})$ and a lateral subdivision (CAl). Most of the fate-mapped cells were observed in the CAm, the main output of the fear circuit (Paré et al., 2004; Ehrlich et al., 2009). Together, our results strongly suggest that at least a portion of the projection neurons of the CA derive from the vLGE. Surprisingly, in Gsx2 mutants the CA did not appear to be significantly altered, at least as marked by the serotonin receptor Htrld. The vLGE is severely reduced in Gs $x 2$ mutants (Stenman et al., 2003a), and as a result, the size of the mutant striatum is reduced by more than one-half (Toresson and Campbell, 2001). Thus, it may be that striatal projection neurons are derived broadly from the vLGE, whereas the CA projection neurons arise from the ventral-most portion of the vLGE, which is spared in Gs $x 2$ mutants (Yun et al., 2001; Stenman et al., 2003c) (Fig. 8).

In the past, the principal neurons of LA, BLA, and CA have been the major focus of fear conditioning studies; however, mounting data indicate that the inhibitory interneurons within this circuit play essential roles in fear-related behaviors (Ehrlich et al., 2009). Many telencephalic interneurons derive from the MGE or CGE and subsequently migrate to populate both subpallial and pallial (i.e., cortical) regions in the telencephalon (Wonders and Anderson, 2006). In fact, fate maps of Nkx2.1-expressing cells show scattered interneurons of the LA, BLA, and CA, most of which are GABAergic ( $\mathrm{Xu}$ et al., 2008). Moreover, embryonic transplantation studies have shown that CGE progenitors, which provide interneurons to the cortex, also contribute neurons to the amygdalar region (Nery et al., 2002). Using the Dlx5/6-cre mice (Stenman et al., 2003a), which induce recombination in most ventral telencephalic derivatives from the MGE, LGE, and CGE, we observed scattered neurons within the LA and BLA (and most other regions of the amygdala). This pattern of recombination differed considerably from the Isll-cre fate maps (i.e., vLGE), where recombined cells were found exclusively in the CA. Thus, the interneuron subtypes found in the amygdalar nuclei likely derive from the MGE, CGE, and/or the dLGE. Within the LA and BLA, we found Dlx5/6-cre fate-mapped cells that coexpressed $\mathrm{CB}$ or $\mathrm{CR}$. Cortical interneurons that express $\mathrm{CB}$ are known to derive from the MGE, whereas many of the CRexpressing interneurons arise in the CGE (Wonders and Anderson, 2006). Both of these interneuron subtypes are GABAergic. Therefore, it seems likely the CB and CR interneurons found within the LA and BLA would have mostly similar origins. Additionally, the preoptic area has been shown to give rise to a specific cortical interneuron subtype (Gelman et al., 2009), and thus it remains possible that this subpallial region may also provide interneurons to the amygdalar complex.

Another subtype of interneurons that populate the amygdalar fear circuit is the ITC. These interneurons are also GABAergic (Marowsky et al., 2005; Jüngling et al., 2008) and are clustered in paracapsular regions on the medial and lateral sides of the basolateral complex. In addition, ITCs congregate at the base of the BLA in the IA. Recent studies have shown that the ITCs are essential for extinction of fearful memories (Jüngling et al., 2008; Likhtik et al., 2008). Despite the recently acknowledged importance of this interneuron subtype for fear-related behaviors, nothing is known about its developmental origins. The ITCs do not express markers typical of cortical interneurons, and thus we suspected that they do not take origin from the MGE or the CGE. Instead, recent data point to the dLGE in the generation of the ITCs. The dLGE marker Tshz1 shows expression along the lateral migratory stream and in paracapsular regions within the amygdala (Caubit et al., 2005). This correlates well with EGFP expression in the Gs $x 2^{E G F P /+}$ mice (Wang et al., 2009), which, we show here, is highly expressed in the dLGE and along the lateral migratory stream as well as in paracapsular regions of the basolateral complex. It is known that the expansion of VP markers in Gs $x 2$ mutants occurs at the expense of dLGE identity (Yun et al., 2001; Stenman et al., 2003c). Thus, it seems likely that the loss of dLGE identity during embryogenesis leads directly to the reduction in ITCs both in the paracapsular region as well as in the IA of Gs $x 2$ conditional mutants, suggesting a dLGE origin for these interneurons. Indeed, the $S p 8$ conditional mutants, which show defects in dLGE development (Waclaw et al., 2006), have similar reductions in the ITCs as those observed in the Gs $x 2$ conditional mutants. It should be noted, however, that not all ITCs are lost in either mutant, suggesting heterogeneity within this population. The dLGE gives rise to a diverse array of olfactory bulb interneurons (Waclaw et al., 2006; Wang et al., 2009). Thus, the dLGE progenitors of ITCs may also exhibit similar heterogeneity. In support of this notion, a recent study has demonstrated both 
functional and anatomical heterogeneity within the ITC population (Geracitano et al., 2007).

The results of this study have allowed us to propose a model of neuronal origins within the amygdalar fear circuit (Fig. 8). Moreover, abnormalities observed in Gsx2 and Sp8 mutants may provide a useful way to examine the behavioral consequences of genetic alterations in the anatomy and connectivity of the mouse fear circuit.

\section{References}

Bielle F, Griveau A, Narboux-Nême N, Vigneau S, Sigrist M, Arber S, Wassef M, Pierani A (2005) Multiple origins of Cajal-Retzius cells at the borders of the developing pallium. Nat Neurosci 8:1002-1012.

Carney RS, Alfonso TB, Cohen D, Dai H, Nery S, Stoica B, Slotkin J, Bregman BS, Fishell G, Corbin JG (2006) Cell migration along the lateral cortical stream to the developing basal telencephalic limbic system. J Neurosci 26:11562-11574.

Carney RS, Cocas LA, Hirata T, Mansfield K, Corbin JG (2009) Differential regulation of telencephalic pallial-subpallial boundary patterning by pax6 and gsh2. Cereb Cortex 19:745-759.

Caubit X, Tiveron MC, Cremer H, Fasano L (2005) Expression patterns of the three Teashirt-related genes define specific boundaries in the developing and postnatal mouse forebrain. J Comp Neurol 486:76-88.

Corbin JG, Gaiano N, Machold RP, Langston A, Fishell G (2000) The Gsh2 homeodomain gene controls multiple aspects of telencephalic development. Development 127:5007-5020.

Ehrlich I, Humeau Y, Grenier F, Ciocchi S, Herry C, Lüthi A (2009) Amygdala inhibitory circuits and the control of fear memory. Neuron 62:757-771.

Ferland RJ, Cherry TJ, Preware PO, Morrisey EE, Walsh CA (2003) Characterization of Foxp2 and Foxp1 mRNA and protein in the developing and mature brain. J Comp Neurol 460:266-279.

Gelman DM, Martini FJ, Nóbrega-Pereira S, Pierani A, Kessaris N, Marín O (2009) The embryonic preoptic area is a novel source of cortical GABAergic interneurons. J Neurosci 29:9380-9389.

Geracitano R, Kaufmann WA, Szabo G, Ferraguti F, Capogna M (2007) Synaptic heterogeneity between mouse paracapsular intercalated neurons of the amygdala. J Physiol 585:117-134.

Hanashima C, Shen L, Li SC, Lai E (2002) Brain factor-1 controls the proliferation and differentiation of neocortical progenitor cells through independent mechanisms. J Neurosci 22:6526-6536.

Hébert JM, McConnell SK (2000) Targeting of cre to the Foxg1 (BF-1) locus mediates loxP recombination in the telencephalon and other developing head structures. Dev Biol 222:296-306.

Hevner RF, Hodge RD, Daza RA, Englund C (2006) Transcription factors in glutamatergic neurogenesis: conserved programs in neocortex, cerebellum, and adult hippocampus. Neurosci Res 55:223-233.

Hirata T, Li P, Lanuza GM, Cocas LA, Huntsman MM, Corbin JG (2009) Identification of distinct telencephalic progenitor pools for neuronal diversity in the amygdala. Nat Neurosci 12:141-149.

Jacobsen KX, Höistad M, Staines WA, Fuxe K (2006) The distribution of dopamine D1 receptor and mu-opioid receptor 1 receptor immunoreactivities in the amygdala and interstitial nucleus of the posterior limb of the anterior commissure: relationships to tyrosine hydroxylase and opioid peptide terminal systems. Neuroscience 141:2007-2018.

Jüngling K, Seidenbecher T, Sosulina L, Lesting J, Sangha S, Clark SD, Okamura N, Duangdao DM, Xu YL, Reinscheid RK, Pape HC (2008) Neuropeptide S-mediated control of fear expression and extinction: role of intercalated GABAergic neurons in the amygdala. Neuron 59:298-310.

LeDoux JE (2000) Emotion circuits in the brain. Annu Rev Neurosci 23:155-184.

Likhtik E, Popa D, Apergis-Schoute J, Fidacaro GA, Paré D (2008) Amygdala intercalated neurons are required for expression of fear extinction. Nature 454:642-645.

Lyons GE, Micales BK, Schwarz J, Martin JF, Olson EN (1995) Expression of mef 2 genes in the mouse central nervous system suggests a role in neuronal maturation. J Neurosci 15:5727-5738.

Marín O, Rubenstein JL (2001) A long, remarkable journey: tangential migration in the telencephalon. Nat Rev Neurosci 2:780-790.

Marowsky A, Yanagawa Y, Obata K, Vogt KE (2005) A specialized subclass of interneurons mediates dopaminergic facilitation of amygdala function. Neuron 48:1025-1037.

McDonald AJ (1982) Cytoarchitecture of the central amygdaloid nucleus of the rat. J Comp Neurol 208:401-418.

McDonald AJ, Mascagni F (2001) Colocalization of calcium-binding proteins and GABA in neurons of the rat basolateral amygdala. Neuroscience 105:681-693.

Medina L, Legaz I, González G, De Castro F, Rubenstein JL, Puelles L (2004) Expression of Dbx1, Neurogenin 2, Semaphorin 5A, Cadherin 8, and Emx1 distinguish ventral and lateral pallial histogenetic divisions in the developing mouse claustroamygdaloid complex. J Comp Neurol 474:504-523.

Millhouse OE (1986) The intercalated cells of the amygdala. J Comp Neurol 247:246-271.

Nakamura T, Colbert MC, Robbins J (2006) Neural crest cells retain multipotential characteristics in the developing valves and label the cardiac conduction system. Circ Res 98:1547-1554.

Nery S, Fishell G, Corbin JG (2002) The caudal ganglionic eminence is a source of distinct cortical and subcortical cell populations. Nat Neurosci 5:1279-1287.

Paré D, Quirk GJ, Ledoux JE (2004) New vistas on amygdala networks in conditioned fear. J Neurophysiol 92:1-9.

Perl AK, Wert SE, Nagy A, Lobe CG, Whitsett JA (2002) Early restriction of peripheral and proximal cell lineages during formation of the lung. Proc Natl Acad Sci U S A 99:10482-10487.

Roozendaal B, McEwen BS, Chattarji S (2009) Stress, memory and the amygdala. Nat Rev Neurosci 10:423-433.

Srinivas S, Watanabe T, Lin CS, William CM, Tanabe Y, Jessell TM, Costantini F (2001) Cre reporter strains produced by targeted insertion of EYFP and ECFP into the ROSA26 locus. BMC Dev Biol 1:4.

Stenman J, Toresson H, Campbell K (2003a) Identification of two distinct progenitor populations in the lateral ganglionic eminence: implications for striatal and olfactory bulb neurogenesis. J Neurosci 23:167-174.

Stenman J, Yu RT, Evans RM, Campbell K (2003b) Tlx and Pax6 co-operate genetically to establish the pallio-subpallial boundary in the embryonic mouse telencephalon. Development 130:1113-1122.

Stenman JM, Wang B, Campbell K (2003c) Tlx controls proliferation and patterning of lateral telencephalic progenitor domains. J Neurosci 23:10568-10576.

Swanson LW, Petrovich GD (1998) What is the amygdala? Trends Neurosci 21:323-331.

Szucsik JC, Witte DP, Li H, Pixley SK, Small KM, Potter SS (1997) Altered forebrain and hindbrain development in mice mutant for the Gsh-2 homeobox gene. Dev Biol 191:230-242.

Toresson H, Campbell K (2001) A role for Gsh1 in the developing striatum and olfactory bulb of Gsh2 mutant mice. Development 128:4769-4780.

Toresson H, Potter SS, Campbell K (2000) Genetic control of dorsal-ventral identity in the telencephalon: opposing roles for Pax6 and Gsh2. Development 127:4361-4371.

Waclaw RR, Wang B, Campbell K (2004) The homeobox gene Gsh2 is required for retinoid production in the embryonic mouse telencephalon. Development 131:4013-4020.

Waclaw RR, Allen ZJ 2nd, Bell SM, Erdélyi F, Szabó G, Potter SS, Campbell K (2006) The zinc finger transcription factor Sp8 regulates the generation and diversity of olfactory bulb interneurons. Neuron 49:503-516.

Waclaw RR, Wang B, Pei Z, Ehrman LA, Campbell K (2009) Distinct temporal requirements for the homeobox gene Gsx2 in specifying striatal and olfactory bulb neuronal fates. Neuron 63:451-465.

Wang B, Waclaw RR, Allen ZJ 2nd, Guillemot F, Campbell K (2009) Ascl1 is a required downstream effector of Gsx gene function in the embryonic mouse telencephalon. Neural Dev 4:5.

Wonders CP, Anderson SA (2006) The origin and specification of cortical interneurons. Nat Rev Neurosci 7:687-696.

Xu Q, Tam M, Anderson SA (2008) Fate mapping Nkx2.1-lineage cells in the mouse telencephalon. J Comp Neurol 506:16-29.

Yun K, Potter S, Rubenstein JL (2001) Gsh2 and Pax6 play complementary roles in dorsoventral patterning of the mammalian telencephalon. Development 128:193-205.

Yun K, Garel S, Fischman S, Rubenstein JL (2003) Patterning of the lateral ganglionic eminence by the Gsh1 and Gsh2 homeobox genes regulates striatal and olfactory bulb histogenesis and the growth of axons through the basal ganglia. J Comp Neurol 461:151-165. 